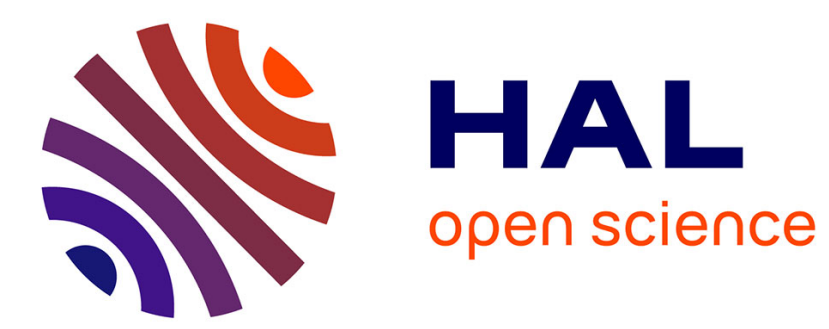

\title{
Les ivresses inattendues du pouvoir local (voyage en ego-politique)
}

\author{
Alain Faure
}

\section{To cite this version:}

Alain Faure. Les ivresses inattendues du pouvoir local (voyage en ego-politique). Sens-Dessous, 2018, Territoires, 21. halshs-01658757

\section{HAL Id: halshs-01658757 https://shs.hal.science/halshs-01658757}

Submitted on 7 Dec 2017

HAL is a multi-disciplinary open access archive for the deposit and dissemination of scientific research documents, whether they are published or not. The documents may come from teaching and research institutions in France or abroad, or from public or private research centers.
L'archive ouverte pluridisciplinaire HAL, est destinée au dépôt et à la diffusion de documents scientifiques de niveau recherche, publiés ou non, émanant des établissements d'enseignement et de recherche français ou étrangers, des laboratoires publics ou privés. 


\section{LES IVRESSES INATTENDUES DU POUVOIR LOCAL (VOYAGE EN EGO-POLITIQUE)}

Introduction. Les mystères du gout du pouvoir 1

LES LARMES QUI PRÉCÈDENT ET ACCOMPAGNENT L'ENTRÉE EN POLITIQUE 2

Blessures et tourments enfantins 3

La première campagne 3

LES PROMESSES QUI FAÇONNENT L'EXERCICE DU POUVOIR

Le regard des autres $\quad 5$

L'ordre et le lieu $\quad 5$

$\begin{array}{ll}\text { Conclusion. Les signes sensibles de la politique, jusqu'à Tokyo } & 7\end{array}$

\section{Introduction. Les mystères du gout du pouvoir}

D’où vient ce mystérieux « gout du pouvoir » qui entraine des individus à s'engager toute une vie dans les arènes électorales et à guerroyer avec tant de ferveur et d'énergie pour « représenter » leurs concitoyens? La réponse à la question est plutôt consensuelle dans la littérature scientifique : les élus courent après la puissance, le prestige, les privilèges, l'autorité et les jeux d'influence. Et dans l'imaginaire collectif, les ascensions politiques sont d'abord affaire de violence, d'argent et de domination, selon un scénario prévisible où la jouissance en politique (la conquête puis la préservation du pouvoir) se nourrit de cris et de sang. Mais dans sa formule restée célèbre, Winston Churchill évoquait aussi les larmes du pouvoir. Cette autre forme d'ivresse politique, qui est moins discutée dans les sciences sociales (hormis chez les historiens, nous y reviendrons) sera au cœur du présent article. Nous souhaitons en effet défendre l'hypothèse que le pouvoir local se nourrit d'abord de blessures, de doutes et de joies, et que c'est dans l'intensité de ces fragilités affectives que l'attrait pour la politique locale se dessine et s'affermit.

L'équation, il faut bien l'avouer, est plutôt mal renseignée dans les enquêtes sociologiques. Pour autant, sa formulation n'est pas tout à fait nouvelle si l'on pense aux travaux de Pierre Clastres dans les années 60 sur les Indiens Guayakis en Amazonie ${ }^{1}$. L'anthropologue avait découvert que le " chef » tirait son autorité d'une fonction d'incarnation du groupe qui ne comportait aucune fonction de commandement. Le leader de la tribu avait même l'interdiction explicite de dicter des règles, de posséder des biens ou d'accéder à des privilèges. Son pouvoir ne s'affirmait, constatait le chercheur avec étonnement, que dans les replis sensibles du langage et du paraître. II tirait sa légitimité de sa capacité à énoncer les valeurs du groupe, valeurs qu'il déclamait sur un mode poétique et onirique et qu'il mettait en scène dans une gestuelle souvent emphatique. Celui qui représentait le collectif n'était que le conteur bavard et légèrement excentrique d'un récit enchanté sur les valeurs et sur l'identité locales. Le groupe lui donnait pour mission d'entretenir par le verbe un rapport esthétique, presque sensuel, à la communauté. Et la coercition était totalement absente des échanges (hormis sur les situations de conflit avec d'autres tribus). La découverte clastrienne a été accueillie avec circonspection dans les arènes académiques et chez les théoriciens du pouvoir. En science politique par exemple, des auteurs "de référence" l'ont réfutée sur le double argument que les résultats ne concernaient que des petites communautés (sur le plan démographique) et que la modernité des Lumières avait entrainé un apprivoisement des passions tandis que Clastres observait des sociétés sans État (ce que ce dernier contestait d'ailleurs en estimant qu'il s'agissait plutôt de sociétés contre l'État). Même si quelques intellectuels ont brillamment prolongé le questionnement (on pense notamment aux travaux du philosophe Miguel Abensour ${ }^{2}$ et de

' Clastres P., 1974, La société contre l'État, Éditions de Minuit.

${ }^{2}$ Abensour M., 2004, La Démocratie contre l'État : Marx et le moment machiavélien, Paris, Le Félin. 
I'anthropologue Marc Abélès ${ }^{3}$ ), la piste du pouvoir non-coercitif organisé contre l'État a été globalement abandonnée en science politique.

Notre propos vise à repérer les larmes, les blessures et les élans de la politique locale contemporaine qui font écho aux travaux de Clastres. Dans les enquêtes que nous avons consacrées aux élus locaux dans plusieurs métropoles du monde, il apparaît que l'accès et l'exercice du pouvoir local se nourrissent d'ivresses non-coercitives. Le matériau d'enquête est constitué par un échantillon de 250 entretiens réalisés en France, en Italie, au Canada et au Japon auprès de personnalités qui ont occupé sur une période longue de leur vie des responsabilités électives de premier ordre au sein des collectivités locales. Nous avons questionné ces leaders sur les raisons qui motivaient leur engagement politique en orientant une grande partie des questions sur leur enfance et leur adolescence. Cette façon de mettre les élus sur le divan ${ }^{4}$, réalisée à partir d'un protocole de recherche standardisé, nous a permis de pointer quelques récurrences dans les réponses qui échappent visiblement aux motifs connus de la puissance et de la domination. Ces données sensibles sont repérables à plusieurs stades: dans le récit d'événements situés avant l'entrée en politique, dans les sentiments liés aux premières expériences électorales, dans la description des tensions et des pressions au quotidien de l'activité politique. Ces données narratives relèvent du sensible, de l'émotionnel, de la psychologie et souvent même presque de la psychanalyse. En centrant l'analyse sur ce matériau hétéroclite, nous avons été confronté à un travail compliqué de codification qui nous a entrainé à strictement anonymiser le contenu des entretiens puis à en extraire les caractéristiques sociopolitiques qui échappaient à la seule explication des traits de personnalité ou à la configuration culturelle.

Au terme de l'enquête (que nous avons volontairement conclue au Japon, pays où l'expression des émotions est particulièrement contenue), il nous semble possible de tirer sur ces données sensibles quelques enseignements à portée générale. Deux types de résultats seront présentés et proposés à la discussion dans cet article. Le premier concerne la politique avant la politique : nous avons repéré une récurrence surprenante, dans les témoignages, concernant l'importance de certaines blessures affectives enfantines et de certaines expériences originelles d'exposition de soi au collectif. Il semble que ces empreintes initiales, invisibles et guère dicibles, pèsent sur les conditions futures d'exercice du pouvoir. Le second enseignement concerne la politique en tension et sous pression : en décryptant les productions narratives des élus, nous avons pu observer la place centrale que les promesses politiques occupaient dans leur activité quotidienne de médiation, que la parole serve à capter la confiance des électeurs, à vanter les atouts du territoire ou à qualifier les dispositifs d'action publique. Cette puissance des mots suggère que l'exercice du pouvoir est indexé à la charge émotionnelle des promesses, et que c'est pour l'ivresse de ces récits enchantés (plus que pour des prérogatives et des privilèges) que les élus locaux investissent si durablement la politique. On se demandera en guise de conclusion dans quelle mesure les récits passionnés sur ces larmes et ces promesses du pouvoir local permettent de repérer une ivresse politique locale universelle jusqu'à Tokyo... C'est l'hypothèse plus générale que les émotions ont un impact sur le jeu démocratique et qu'il faut prendre au sérieux l'ego-politique de la politique, à la fois comme résultat et comme méthode d'analyse.

\section{LES LARMES QUI PRÉCÈDENT ET ACCOMPAGNENT L'ENTRÉE EN POLITIQUE}

En interrogeant les élus sur leur vie avant la politique, nous avons été surpris par l'intensité des témoignages recueillis alors même que les premiers souvenirs n'étaient pas perçus comme en lien avec l'évocation de leur future vocation. Les enfants portent le plus souvent sur la politique un regard d'ahurissement et d'incompréhension, un monde, celui des adultes, où les interactions sont violentes et mystérieuses, bref une activité énigmatique (c'est une source soudaine de violence) et pleine de tabous (personne ne l'explique ni ne l'enseigne). La politique est opaque pour l'enfant mais elle surgit dans les mémoires autour de quelques séquences précises qui véhiculent toujours une forte charge émotionnelle. Aucun des élus rencontrés n'a eu la " révélation " qu'il serait plus tard un élu mais tous racontent qu'ils ont été intrigués et souvent captivés par les passions et les tensions qu'ils pressentaient derrière le qualificatif " politique ". C'est cette empreinte émotionnelle qui devient, au fil des témoignages, un résultat en tant que tel : le futur élu conserve une perception aiguë des premiers instants où il a pris conscience de ce qu'il traduira par la suite comme des enjeux de pouvoir. Au début, il s'agit juste de signaux inquiétants et visiblement importants à prendre en considération. La plupart des futurs élus décrivent un mécanisme puissant d'attirance et de répulsion qui fonctionne comme un révélateur : la politique ne laisse personne indifférent, son rôle est

\footnotetext{
${ }^{3}$ Abélès M., 2014, Penser au-delà de l'État, Paris, Belin.

${ }^{4}$ Faure A., 2016, Des élus sur le divan. Les passions cachées du pouvoir local, Presses Universitaires de Grenoble.
} 
certes opaque mais ses effets paraissent immenses. Si l'on distingue dans les témoignages une grande diversité de situations qui caractérisent cette séquence de découverte, il est possible de pointer des récits récurrents sur les deux empreintes des drames de l'enfance et du premier engagement électoral.

\section{Blessures et tourments enfantins}

A la question "Quelle est la première émotion, dans l'enfance, qui pourrait faire écho à votre attrait pour la politique?", les réponses prennent d'abord un tour connu. Sont souvent évoqués les tourments lorsque l'enfant perçoit un conflit entre ses parents (sur des valeurs, sur des clivages idéologiques ou culturels, sur des histoires territoriales différenciées). Il y a aussi dans l'atmosphère familiale des ingrédients politiques troublants au moment des élections ou pour qualifier des positions sociales dans les repas de famille. Les grands parents et les oncles et tantes, la fratrie, la religion, voilà autant de sujets où la politique colore parfois fortement les propos et impressionne les enfants, surtout dans leur soudaine tonalité conflictuelle. Les trajectoires familiales constituent un espace codifié où se forgent les premières émotions politiques, ce n'est pas une surprise. La filiation directe ou symbolique occupe par exemple une place non négligeable sur cette séquence même si l'enquête montre surtout une grande diversité de parcours. À coté des héritiers $(20 \%$ de l'échantillon), on trouve surtout des capacitaires et des leaders en herbe. .Les témoignages révèlent sur cette séquence un résultat inattendu concernant la place décisive de certaines influences et de certains événements. On pense par exemple à la figure du père : dans $80 \%$ des récits, l'image paternelle est fébrilement perçue par l'enfant en lien avec des questions d'autorité et de pouvoir. La figure est même omniprésente dans certaines régions, que ce soit en admiration ou en opposition. A Naples par exemple, il semble presque impossible pour un élu de parler politique sans raconter sur un mode quasi-psychanalytique son " rapport au père " et l'ivresse démesurée que la politique occupe dans cette relation.

De même, nous avons recueilli une quantité surprenante de souvenirs qui concernent des traumatismes et des blessures. Là où l'on attendait plutôt des enfances heureuses et des success stories (au vu des biographies consultées), les futurs élus nous ont souvent raconté des enfances jalonnées de drames, d'écorchures et de révoltes. Nos interlocuteurs disent tous l'hyper sensibilité de leur regard d'enfant sur les inégalités et les injustices qui les entourent. Les récits empruntent aussi très souvent les sentiers de l'échec ou de l'enfermement pour décrire le monde. Les récits sur la scolarité ravivent par exemple des perceptions contrastées comme celles de l'enfant turbulent, incompris ou délaissé. On trouve aussi dans les témoignages, de façon itérative, la description de moments difficiles qui ont marqué la socialisation politique. Dans bien des récits, la maladie et la mort (affrontées dans l'environnement proche de l'enfant et de l'adolescent) constituent le déclencheur de la prise de conscience que la politique possède une fonction majeure : elle est là pour tenter de changer les injustices du monde. Plus classiquement, les témoignages regorgent aussi de rencontres marquantes (des personnalités qui ont ébloui) et d'événements perçus comme fondateurs (une manifestation, une grève, une tragédie).

Pour toutes tous ces empreintes, c'est la charge émotionnelle qui retient l'attention : chez l'élu, la prise de conscience de la politique s'est faite " à vif » et que cette forme de mise en danger était vécue sur un mode tourmenté et douloureux. Le " petit » enfant (qui deviendra un " grand » élu) retient ses larmes mais la souffrance est palpable dans les récits. Parfois même, l'entretien permet de mettre à jour des données sensibles qui relèvent presque du transfert sur le plan psychanalytique. Nous avons vu certains interlocuteurs perdre pied et pleurer pendant l'entretien. Ces indices sont, pour notre enquête, des données difficiles à analyser mais impossibles à passer sous silence en raison de leur récurrence et de leur intensité.

\section{La première campagne}

Une autre trace des empreintes initiales s'est dessinée sur la séquence des premiers engagements du futur élu dans une arène électorale. Il existe sur cette question des travaux approfondis qui détaillent les mécanismes de transmission, de filiation et de reproduction du pouvoir. Les données sociopolitiques nous informent sur une éligibilité construite par des règles propres aux représentations du pouvoir dans chaque territoire. Mais, et c'est la seconde surprise de notre enquête, on découvre au fil des témoignages que cette entrée en politique se réalise toujours au filtre d'une expérience émotionnelle singulière. En première lecture, les élus nous expliquent presque systématiquement qu'ils sont entrés en politique " par hasard » et que leur histoire ne pouvait guère être généralisée tant leur parcours était « atypique ». Cette façon de se percevoir en décalage avec les professionnels de la politique (même lorsque les élus sont à l'évidence des héritiers ou des leaders en herbe) nous renseigne sur une donnée que les sociologues ont souvent passé sous silence: la 
première expérience électorale est toujours décrite a posteriori comme une épreuve et comme une exposition de soi imprévue et sans équivalent. Les émotions y occupent une place centrale : les candidats ont ressenti à cette occasion un frisson (le danger, l'aventure, le charisme...) dont ils n'avaient pas imaginé la saveur.

Les descriptions de la première campagne insistent toutes sur la tonalité fiévreuse de cette exposition aux autres. En allant pour la première fois « au combat » et " sur le terrain », les futurs élus vont de découverte en découverte, ils estiment -rétrospectivement- que le moment était enivrant, grisant, exaltant, unique. Ils gardent intacts des détails mémorables sur le travail d'équipe, les premiers tracts, les slogans de campagne, les réunions publiques, la densité des contacts directs avec la population, le décomptage fébrile autour des urnes. Ils évoquent aussi volontiers certaines rencontres qui se sont avérées par la suite décisives, que ce soit avec un baron prestigieux, un mentor pris pour modèle ou une équipe de campagne particulièrement attachante. Parfois enfin, ils reconnaissent que c'est à ce moment précis qu'ils ont pris conscience du plaisir qu'ils éprouvaient à faire de la politique, à parler en public, à mener une troupe, à tisser des alliances, à collecter des soutiens, à chercher des solutions, à interpeler des adversaires... Les récits convergent d'ailleurs tous sur ce point : qu'ils aient gagné ou perdu ce premier défi électoral, c'est dans la fièvre de cette expérience qu'ils ont attrapé le virus de la politique, dans l'intensité de cette soudaine exposition au regard et à l'écoute des autres.

Les confidences sur les émotions politiques enfantines et les témoignages sur l'entrée en politique nous renseignent donc sur des dimensions méconnues de l'engagement politique. Avant d'accéder au pouvoir et de l'exercer durablement, l'éligible territorial est certes celui qui, au sens anthropologique du terme, détient certaines qualités sociopolitiques qui en font un élu potentiel sur un territoire précis. Mais cette éligibilité ne prend chair et consistance que sur le mode sensible d'une surexposition affective, dans l'entremêlement d'une profusion de sentiments d'inquiétude, de désir et de jubilation. Les souvenirs enfantins et la mémoire de la première campagne semblent d'autant plus vifs qu'ils sont nourris de morsures affectives. Le constat fait écho à ce que les historiens nomment parfois une colère de vérité, quand les individus construisent leur rapport au monde au tamis d'appréhensions intimes, violentes et égocentrées. Et l'on retrouve ensuite nombre de ces empreintes émotives dans la façon qu'ont les élus de faire de la politique par la suite. On pense par exemple au lien qui relie certains drames vécus dans l'enfance et la peau de crocodile des élus de premier niveau dans le combat politique. On pense aussi à la fulgurance des amitiés indélébiles tissées avec quelques proches dans les premiers combats politiques. La fidélité et la longévité en politique sont deux énigmes que ces données éclairent. C'est le premier résultat inattendu de notre enquête : les larmes qui précèdent et accompagnent l'entrée en politique s'apparentent à des ivresses structurantes, au sens où elles permettent de mieux comprendre la détermination hors du commun qui caractérise ensuite l'engagement politique.

\section{LES PROMESSES QUI FAÇONNENT L'EXERCICE DU POUVOIR}

Une seconde énigme du gout du pouvoir concerne l'énergie que les élus locaux déploient au quotidien de leur mandat, au fil de journées interminables et dans l'enchainement sans répit des contacts interpersonnels, des réunions publiques, des déplacements et des micro-séances de travail. Cette activité a fait récemment l'objet d'études quantifiées qui montrent à quel point leurs agendas sont sursaturés ${ }^{5}$. Pour autant, peu d'études questionnent les ressorts de cette boulimie relationnelle autrement que sur l'argument tautologique du "gout du pouvoir ». Dans les tournois compétitifs et incertains où les élus sans cesse s'exposent et se confrontent, les prises de parole montrent des jeux collectifs où les joutes se nourrissent d'affrontements, de bravades, d'euphorie, de fierté, mais aussi d'impuissance et de solitude.

Au cours des entretiens, nous avons cherché à comprendre comment les élus parvenaient à construire leur équilibre dans cette effervescence affective, comment ils gardaient un cap malgré la vigueur des sollicitations et la rugosité des polémiques au quotidien de leur activité. Une partie de l'explication se trouve peut-être, une fois encore, du côté des découvertes de Pierre Clastres lorsque ce dernier étudiait la transcendance des discours du chef en sa tribu. L'anthropologue constatait que dans les communautés étudiées, l'adrénaline du pouvoir se logeait d'abord dans la magie des mots que le chef utilisait pour donner corps aux valeurs de la tribu. Le leader était celui qui tirait sa légitimité (et qui se singularisait du groupe) grâce à sa capacité à raconter une histoire commune, à formuler des promesses et à suggérer un horizon collectif. Et en mettant des mots sur l'existence du groupe, il partageait l'expérience sensible d'un épanouissement et d'une ivresse.

${ }^{5}$ Godmer L., Marrel G., 2016, La politique au quotidien. L'agenda et l'emploi du temps d'une femme politique, Lyon, ENS 
La piste mérite d'être discutée à nouveau à l'écoute des élus locaux que nous avons rencontrés. Ces derniers développent en effet une aptitude langagière et une compétence communicationnelle qui rappellent cet épanouissement et cette ivresse. En permanence, dès qu'ils prennent la parole, ils cherchent les gestes et les mots justes du territoire (nous y reviendrons plus loin). Nous avons souvent été impressionné, dans les entretiens, par cette attention particulière qu'ils portaient à la qualité et à la justesse de la transaction. Parler politique au nom du groupe, c'est d'abord inviter ses interlocuteurs sur les registres de la sincérité et de l'authenticité. Que l'élocution soit volontariste, tâtonnante, saccadée ou liante, le ton vise toujours une esthétique de l'argumentation. L'alchimie du discours vise souvent deux objectifs : ne pas perdre la confiance des électeurs et énoncer "l'ordre des choses " propre au territoire d'élection.

\section{Le regard des autres}

Le souci de gagner et de conserver la confiance des électeurs n'est pas une surprise. Mais nous avons constaté que cette préoccupation se traduisait par une rhétorique moins attendue : sans cesse, les élus locaux expliquent leur volonté de se situer dans le faire. Ils racontent volontiers, au cœur des tensions et des négociations qui remplissent leur mandat, comment ils intercèdent, interviennent et prennent des décisions. Le propos pourrait faire sourire quand on sait combien l'image du notable reste plutôt attachée, dans l'imaginaire politique, à des "qualités " de temporisation et d'apaisement. Mais la nécessité d'agir de façon concrète et pragmatique constitue bien, dans tous les témoignages, une intention première de l'engagement politique. La confiance avec les électeurs se jauge, nous disent-ils, à l'aune de cette obligation, que la responsabilité politique porte sur une collectivité de 500 ou de 500000 individus. Différentes déclinaisons de ce mode $d u$ faire sont avancées: prendre les "bonnes" décisions pour le collectif, trouver les "bons" interlocuteurs, "garder le contact» (avec les électeurs et avec la réalité de terrain), être à l'écoute, comprendre les attentes, savoir les traduire...

L'obligation du contrat de confiance s'apparente à une figure obligée: les élus locaux sont les dépositaires d'un destin collectif qui se construit à partir d'une relation personnalisée avec les électeurs. L'exercice exige une forme d'empathie émotionnelle dans laquelle l'élu reconnaît qu'il est redevable des promesses qu'il formule. Il se perçoit comme un obligé de ceux qu'il représente et il accorde énormément de crédit à la façon dont on juge son action. Il y a une tension dans cette dette qui envahit et obsède tous les élus locaux. L'équation est fort bien mise en image dans le film-documentaire qu'Yves Jeuland a consacré en 2013 au maire de Paris ${ }^{6}$. Au terme de plusieurs heures d'entretiens, le réalisateur a mis en scène un Delanoé libéré qui semble aimanté par ses électeurs et sans cesse bousculé par cette ardente relation de confiance. Dans un décor épuré (seul face à la caméra), il se confie sur quarante ans d'engagement avec une sincérité désarmante: tour à tour fragile et intouchable, obnubilé par le regard des Parisiens et insensible aux contestations, proche et distant des autres, attentionné et froid, rempli de certitudes et rongé par les doutes, orgueilleux et complexé... Le film révèle admirablement une dimension de la politique qui semble très difficile à décrire et qui a presque toujours imprégné les entretiens que nous avons recueillis : la fièvre politique relève d'une essence où s'entremêlent la fierté et le doute, la supériorité et l'humilité, l'assurance et les fêlures. Les discours récurrents sur la capacité à agir construisent cette tension émotive et ce tournis singulier. Le regard des autres devient obsessionnel : sans cesse, il nourrit un tourment qu'il faut canaliser par le verbe.

"Les mots font violence aux choses ", écrivait Michel Foucault pour qualifier la puissance invasive du langage dans les jeux de pouvoir ${ }^{7}$. On perçoit avec acuité dans ce documentaire comment les promesses politiques sont l'expression première de cette violence symbolique : elles permettent aux élus de figurer à la fois l'action et la représentation, la puissance et l'empathie.

\section{L'ordre et le lieu}

L'autre source d'adrénaline du pouvoir local concerne les discours sur l'identité locale. L'élu local est sommé en permanence par son entourage d'assumer une fonction de représentation au sens spatialisé du terme: on attend de lui, en toute occasion, qu'il incarne le territoire dans son épaisseur physique et sociale. Quelle que soit la taille de collectivité, il doit raconter l'espace qu'il gouverne, il doit montrer qu'il est habité par le lieu, qu'il en connaît l'histoire, qu'il en maitrise les rouages administratifs, qu'il en éprouve l'esprit et même qu'il n'en sous-estime pas certains mystères. A chaque nouvelle rencontre avec un individu ou un groupe, il doit évoquer cet imaginaire et en faire le récit. Dans sa façon de s'exprimer, cette injonction à la

\footnotetext{
${ }^{6}$ Jeuland Y, 2013, Delanoé libéré, France 3, 50 mn (diffusé le 18/10/2013).

${ }^{7}$ Foucault M., 1966, Les mots et les choses. Une archéologie des sciences humaines, Paris, Gallimard.
} 
territorialité génère ce que le politiste Zittoun nomme des lignes narratives génériques ${ }^{8}$. Nous en avons repéré deux au fil des témoignages.

La première concerne la rhétorique de l'ordre local des choses. Avec les grandes lois de décentralisation votées depuis quelques décennies (partout dans le monde), les collectivités locales se transforment en professionnalisant leurs structures et leurs missions. Les nouvelles responsabilités liées aux transferts de compétences touchent tous les domaines de l'action publique. L'élu local «major » est dorénavant un décideur entouré de hauts fonctionnaires et conseillé par des experts. II fait des politiques publiques sur un mode entrepreneurial qui peut rappeler l'activité d'un ministre ou d'un directeur d'administration. L'évolution fait d'ailleurs l'objet de nombreux travaux en science politique et en sociologie politique. Pour qualifier le tournant néo-libéral des années 90 , les politistes qui questionnent la place et le rôle des élites politiques locales portent le plus souvent un regard critique sur les processus de mimétisme et de standardisation à l'œuvre 9 . C'est l'idée que l'ordre des choses se construit et se régule dorénavant en grande partie dans des arènes compétitives à la fois extra-territoriales et extra-publiques. Mais d'autres auteurs insistent aussi sur un phénomène de glocalisation dans la régulation publique qui brouille les cartes de l'articulation entre le public, le privé et la société civile ${ }^{10}$. On retrouve bien les signes de cette hybridation incertaine dans la façon dont les élus locaux appréhendent leur rôle de décideur. Les discours vantent volontiers les vertus du bon sens et de l'imagination, comme s'il fallait conjurer les raisonnements bridés par des priorités gestionnaires ou managériales. L'élu local cherche aussi à résister à l'uniformisation des dispositifs en décrivant sa collectivité comme un écosystème particulier, un espace de négociation fragile dont il faut contenir les tensions et favoriser les expérimentations. L'élu argumente ce que les sociologues nomment une expertise situationnelle et localisée : il mobilise les mots valises du pouvoir (gouvernance, développement durable, éco-citoyenneté, transition, rigueur budgétaire...) en les utilisant sur un mode différencié. II manipule sans cesse la grammaire des politiques publiques pour que le récit soit politiquement audible et localement acceptable.

La seconde ligne narrative concerne la façon sensible de raconter l'esprit des lieux, de lier dans un même récit l'identité et les valeurs du territoire. Une facette de cette alchimie a déjà été dévoilée par un sociologue américain, Mark Kesselmann, à l'étude du pouvoir dans les communes rurales en France dans les années $60^{11}$. Ce dernier a bien montré comment le maire s'octroyait un rôle de chef d'orchestre pour imposer par le verbe un consensus ambigu. Ses plaidoyers revendiquent l'harmonie naturelle mais c'est sa baguette qui impose le tempo et qui guide avec autorité la partition, au profit parfois exclusif de quelques groupes dominants sur le territoire. Cinquante ans plus tard, la métaphore reste efficace pour repérer les acteurs et les groupes d'intérêts qui captent le pouvoir local par le langage. Une littérature abondante se développe par exemple pour dévoiler les enjeux de domination dans les gouvernements intercommunaux, en milieu urbain comme en milieu périurbain ou rural. Cependant, il nous semble que la magie du verbe opère aussi à l'insu des leaders politiques, au sens où ces derniers sont souvent agis par la collectivité : ils n'ont d'autre choix que de raconter l'harmonie communautaire et de lister les dimensions identitaires de cet ordre local des choses. Ici encore, on constate que les propos s'accompagnent toujours d'une forte charge émotionnelle. Ils nous racontent un lien viscéral à l'identité. Le discours des élus sur les racines du territoire fait penser à ce ce que I'historien Yvan Jablonka nomme "la tension vers la réalité »: énoncer son rapport au territoire, c'est immédiatement dire des choses essentielles, c'est s'exposer sur son enfance, sa famille, son quartier, sa religion, son école, ses paysages... Ces récits sont puissants parce qu'ils évoquent à la fois la profondeur (l'enracinement, l'ancrage, la fidélité, le patrimoine) et l'horizontalité (la proximité, le voisinage, les solidarités, le réseau).

La confiance, l'ordre et le lieu sont trois ingrédients qui reviennent en boucle dans les discours des élus locaux. La charge émotionnelle qui accompagne leur évocation suggère que l'engagement politique se nourrit de cette adrénaline. Des questionnements sensibles, presque existentiels, s'y révèlent: être à la hauteur du regard des autres, incarner les valeurs du territoire, raconter une identité collective... Dans ses déclinaisons quotidiennes, la médiation politique locale entraine une forme d'ivresse où ce sont ces promesses, plutôt que des combats, qui façonnent l'exercice du pouvoir.

\footnotetext{
${ }^{8}$ Zittoun P., 2013, La fabrique politique des politiques publiques, Paris, Presses de Sciences Po,

${ }^{9}$ Jobert B., 1994, Le tournant néolibéral en Europe, Paris, L'Harmattan.

${ }^{10}$ Muller P., 2015, La société de l'efficacité globale, Paris, PUF.

${ }^{11}$ Kesselman M., 1972, Le consensus ambigu. Étude sur le gouvernement local, Grenoble, Cujas.
} 


\section{Conclusion. Les signes sensibles de la politique, jusqu'à Tokyo}

Dans quelle mesure les données sensibles que nous avons pointées au fil de nos enquêtes constituentelles des invariants du pouvoir local? Dans les témoignages recueillis, on trouve à chaque fois beaucoup d'intensité émotionnelle dans les propos. Raconté à la première personne, la politique dévoile une facette sensible plutôt inattendue où les larmes et les promesses précédent souvent le sang et la violence. La politique changerait-elle de nature quand on la décrypte de l'intérieur, quand on la retrace au filtre intime de chaque trajectoire de vie? Un questionnement un peu comparable a été exploré il y a 50 ans par Paul Veynes avec sa réflexion théorique sur l'historien comme narrateur et romancier $d u$ vrai ${ }^{12}$. Son objectif était double: d'une part regarder le passé en restituant la façon sensible dont les acteurs le vivaient et d'autre part questionner jusqu'à l'auto-analyse les grilles de lecture adoptées par ceux qui travaillent sur la mémoire. Les historiens qui ont par la suite prolongé ce courant d'analyse ont toujours veillé à ne pas dissocier les données sociohistoriques de leur appréhension intime. Ils ont cherché en quelque sorte à combiner le regard clinique du scientifique et celui plus littéraire de l'écrivain. Un chercheur contemporain, Ivan Jablonka, illustre avec brio cette démarche hybride où la description des trajectoires de vie intègre en permanence des donnes sensibles qui sont toujours situées et contextualisées ${ }^{13}$. Dans notre enquête auprès des élus locaux, nous nous sommes inspirés de cette sensibilité à l'ego-histoire pour faire en quelque sorte de l'ego-politique. Les souvenirs enfantins, la mémoire des premières campagnes électorales et le tournis de l'exercice du pouvoir au quotidien ont été considérés comme des données à part entière. Elles nous ont permis de relier potentiellement le mystère de l'engagement politique à des empreintes émotives structurantes. Et en faisant parler les élus sur leur mandat, nous avons pu repérer des vecteurs d'adrénaline (le regard des autres, la magie des lieux, l'ivresse des promesses...) qui façonnaient visiblement l'exercice du pouvoir au quotidien.

Tous ces indices sensibles plaident, nous semble-t-il, pour prendre au sérieux l'entrée par l'egopolitique. Notons que la notion a déjà été utilisée en 2013 par Christian Le Bart pour décrire des élus locaux en campagne ${ }^{14}$. Dans une période caractérisée par une individualisation croissante du champ politique, le politiste constate que les candidats s'exposent de plus en plus ouvertement aux électeurs, sans filtre et de façon intimiste, et qu'ils surjouent souvent dans leur communication les cartes de l'empathie et de la proximité. La médiation politique prend une tournure personnalisée et émotive en rupture avec les expertises professionnalisées. On peut élargir la définition de Christian Le Bart en inscrivant l'ego-politique dans deux dimensions. La première est descriptive et inductive. L'ego-politique est la façon sensible que les individus adoptent pour vivre les enjeux collectifs. Il s'agit ici d'orienter la focale sur les épreuves émotives qui imprègnent leurs représentations de la politique, que ce soit dans l'enfance et l'adolescence ou dans la pratique concrète du pouvoir. Dans cette perspective, l'ego-politique de l'élu local, ce sont les larmes et les promesses qui précèdent, qui accompagnent et qui formatent son attrait pour la politique, l'autorité et le pouvoir. La seconde façon de poser les problèmes consiste à considérer, à l'instar des historiens avec l'egohistoire, que I'ego-politique est une posture d'analyse, critique et historicisée, qui situe toujours les diagnostics sur la démocratie en explicitant d'où l'observateur observe, avec quelle sensibilité analytique et à partir de quel protocole de recherche. Sur l'énigme du gout du pouvoir local (c'était notre point de départ), ces deux focales apportent chacune leur lot d'explications et de points d'interrogation.

Sur son versant descriptif, l'entrée par l'ego-politique permet de faire l'hypothèse que les ivresses du pouvoir local sont de même nature dans tous les parties du monde où nous avons enquêté. C'est assurément le résultat le plus inattendu de la recherche. En la terminant par six mois d'immersion au Japon, nous souhaitions éprouver la portée des résultats dans un pays où la place des émotions dans la politique est particulièrement difficile à saisir. Roland Barthes a certes montré que l'harmonie d'ensemble se dessinait, s'imposait et se fissurait dans la délicatesse avec laquelle chaque individu en manipulait et en interprétait les signes $^{15}$. Mais on sait aussi qu'au Japon, toute expression personnalisée des émotions est bannie de la scène politique, notamment sur la conviction que la quête de vérité n'implique jamais le sujet et que les élus incarnent ce déni des affects personnels (déni que l'on retrouve jusque dans la sphère privée, seule nation occidentale où la psychanalyse n'existe pratiquement pas en tant que thérapie...). Dans les entretiens menés en tête à tête (dans leur langue maternelle et sous le sceau de la confidentialité), les élus rencontrés ont certes affiché une extraordinaire impassibilité corporelle mais à notre grande surprise, ils ont tous, sans exception,

\footnotetext{
${ }^{12}$ Veynes P., 1971, Comment on écrit l'histoire : essai d'épistémologie, Paris, Seuil.

${ }^{13}$ Jablonka I., 2012, Histoire des grands-parents que je n'ai pas eus, Paris, Seuil ; Jablonka I., 2014, L'histoire est une littérature contemporaine, Paris, Seuil.

${ }^{14}$ Le Bart C., 2013, L'ego-politique. Essai sur l'individualisation du champ politique, Paris, Armand Colin.

${ }^{15}$ Barthes R., 1970, L'empire des signes, Paris, Flammarion.
} 
brisé la glace des conventions formelles en acceptant de décrire très explicitement les raisons profondes de leur attrait pour la politique. Sur les empreintes de l'enfance par exemple, nous avons recueilli des confidences intimistes étonnantes de sincérité concernant des drames familiaux et des souffrances enfantines. Sur le récit des campagnes électorales, la même adrénaline que celle rencontrée à Naples ou à Montréal marque les premières expériences (comme le récit récurrent de cette séquence, incongrue pour nous, où le candidat se retrouve à l'entrée des gare, un mégaphone à la main et dans l'indifférence générale, pour présenter son programme). Ces résultats ressemblent beaucoup à ce qui a été observé dans nos précédentes enquêtes. Une fois surmonté le léger effroi de la première question (avec parfois un long silence, mais la courtoisie obligeait toujours notre interlocuteur à ne pas éviter les questions), le jeu de la sincérité et du dévoilement a fonctionné à plein dans les entretiens, que ce soit pour décrire les blessures des premières armes en politique, pour commenter le consensus trompeur du combat politique (avec l'obsession du regard des autres) ou encore pour mettre en discours une véritable esthétique de l'ordre (avec des plaidoyers enflammés sur le génie des lieux). Les résultats de cette immersion japonaise suggèrent donc une forme d'universalité dans les façons de ressentir et de vivre les passions du pouvoir local. Comme à Naples (où l'on attendait plutôt le sang du clientélisme et de la corruption), comme à Montréal (où l'on nous annonçait la main mise cynique des " gens d'affaire »), comme dans les collectivités locales en France (où c'est la figure du notable stratégiquement placé à la croisée des intérêts qui était censée résumer le gout du pouvoir).

Sur son versant analytique, ces résultats alimentent un questionnement balbutiant en sciences sociales (hormis chez les historiens ${ }^{16}$ ) sur la place des émotions dans la vie sociale. En nous inspirant de travaux stimulants de sociologie politique ${ }^{17}$, nous avons récemment tenté d'ouvrir une controverse collective sur la politique à l'épreuve des émotions dans le champ éclaté des sciences du politique ${ }^{18}$. Les auteurs y discutent chacun à leur façon l'hypothèse d'un tournant émotionnel dans les façons de faire de la politique (comme on a pu débattre dans les années 80 d'un tournant linguistique ou plus récemment d'un tournant territorial). La démocratie sensible en est encore, sur le plan analytique, au stade de la terra incognita. Notre recherche sur les élus locaux alimente de chantier en montrant que l'engagement en politique s'accompagne d'une forme singulière d'euphorie (presque d'ébriété) qui combine plusieurs ivresses. A titre exploratoire, il nous semble possible de spécifier cette diversité sur trois registres distincts. Le premier concerne le respect de l'autorité de l'État et la fameuse servitude volontaire théorisée par Etienne de La Boétie. L'apprivoisement des passions ${ }^{19}$ semble moins évident depuis quelques décennies, comme si la charge émotive des arguments contre l'État hégémonique gagnait du terrain. Le deuxième concerne la magie des lieux, rhétorique dont les leaders politiques locaux se saisissent souvent dans leurs discours pour faire le récit fervent d'un horizon collectif qui serait micro-territorial plutôt que national. Le troisième registre enfin concerne les aspirations à la citoyenneté politique qui s'affichent dans les réseaux sociaux et dans les arènes participatives, et dont les élus locaux seraient les garants naturels. On y entrevoit un droit au bonheur sans limite et une esthétique égocentrée qui rappellent la philosophie des passions (tristes et joyeuses) chère aux spinozistes ${ }^{20}$. L'État contesté, la magie des lieux et la citoyenneté du nombril sont trois énigmes contemporaines pour les théoriciens de la domination. Rapportées à l'exercice du pouvoir local, ces énigmes nous incitent à discuter les travaux de référence sur le consentement (des individus), la sujétion (au pouvoir) et le contentement (citoyen). Le voyage en ego-politique propose, dans cette perspective, des clefs de lecture stimulantes. Y compris, bien sûr, sur les ivresses et les aveuglements du chercheur dans son rapport sensible à la question démocratique...

Alain Faure - Directeur de recherche CNRS, Pacte, Sciences Po Grenoble, Université Grenoble Alpes Carnet de recherche : http://enigmes.hypotheses.org/

\footnotetext{
${ }^{16}$ Corbin A., Courtine J.-J., Vigarello G., 2017, Histoire des émotions, De la fin du XIXe siècle à nos jours, Paris, Seuil.

${ }^{17}$ Braud P., 1971, Le jardin des délices démocratiques, Paris, Presses de la FNSP; Traïni P., 2009, Emotions... Mobilisation!, Paris, Presses de Sciences Po.

${ }^{18}$ Faure A., Négrier E. dir., 2017, La politique à l'épreuve des émotions, Rennes, Presses Universitaires de Rennes.

${ }^{19}$ Elias N., 1939, La civilisation des mours, Paris, Presses Pocket.

${ }^{20}$ Lordon F., 2016, Les affects de la politique, Paris, Seuil.
} 